\title{
Brane Probes, Toric Geometry, and Closed String Tachyons
}

\author{
Tapobrata Sarkar * \\ the Abdus Salam \\ International Center for Theoretical Physics, \\ Strada Costiera, 11 - 34014 Trieste, Italy
}

\begin{abstract}
We study non-supersymmetric orbifold singularities from the point of view of D-brane probes. We present a description of the decay of such singularities from considerations of the toric geometry of the probe branes.
\end{abstract}

*E-mail: tapo@ictp.trieste.it 


\section{Introduction}

In recent years, much attention has been paid to the study of tachyonic instabilities in string theory. These instabilities, which usually arise in the absence of space-time supersymmetry, have received great interest both in the context of open and closed strings. For open string theories, tachyonic instabilities have been studied widely, following the pioneering work of Sen [1]. Closed string tachyons have also received attention of late, following the work of Adams, Polchinski and Silverstein (APS) [2]. Whereas open string tachyon condensation can be studied in the boundary state formalism, and usually leads to a change in the brane configuration (for eg. annihilation or decay of D-branes), closed string tachyon condensation leads to a decay of the space-time itself. This in itself can be a difficult problem to study, but there is a class of examples that can be analysed with known methods. These are the non-supersymmetric orbifolds, with localised tachyonic instabilities, first studied in [2].

Resolution of supersymmetric orbifolds in closed string theory has been studied in great details in the past. (see, for eg. [3]). In the context of open string theories, i.e using D-branes as probes of these orbifolds, a much richer structure emerges (for a review, see [四). The papers [2], [5], [6] discusses the application of these techniques to non-supersymmetric orbifolds with tachyonic instabilities, with fundamentally new consequences (See also [7] and references therein).

In the typical examples studied in [2], [5] and [6], the action of the nonsupersymmetric orbifold breaks space-time supersymmetry. Demanding that the (tachyonic) instabilities herein are localized at the orbifold fixed point, one can track the behaviour of the orbifold theory with the decay of these instabilities. It was found in these works, that the decay of the localised tachyonic instability usually drives the non-supersymmetric orbifold to a supersymmetric configuration. In [2], this issue was studied using D-brane probes of such orbifolds. Considering the world volume gauge theory of a D-brane that (lives in the transverse space of the orbifold and) probes the singularity, one can, using quiver diagram techniques developed by Douglas and Moore [8], follow the modification of the gauge theory as the tachyon condenses, and hence track the behaviour of the orbifold under tachyon condensation.

In [5], this issue was considered by Vafa, who studied RG flows of the closed string world sheet linear sigma model [9]. This was done for both compact and non-compact orbifold examples, using the mirror description of these models 
[10]. The analysis therein clearly validates the flow patterns discussed in [2], and provides a powerful sigma-model tool to study the same. In [6], Harvey, Kutasov, Martinec and Moore (HKMM) have presented a method to analyse non-supersymmeric orbifolds, using chiral ring techniques and the dynamics of NS 5-branes in the dual picture. Their method consists of the study of the chiral ring structure of the $N=2$ superconformal field theory of the closed string world sheet. Using the direct correspondence of the chiral ring structure of the world sheet SCFT to the geometric resolution of orbifold singularities, the HKMM method is to study the deformation of the chiral ring along the tachyon condensation and hence derive from it the fate of the initial singularity along the world sheet RG flow effected by the tachyon condensation. For nonsupersymmetric orbifolds, HKMM defined the quantity $g_{c l}$, the coefficient in the expression of the asymptotic density of states in the CFT, and conjectured that this decreases along the RG flow, while leaving the effective central charge of the theory unchanged.

It is of interest to continue these investigations along the lines of APS, Vafa and HKMM, to understand the more general underlying structure of these nonsupersymmetric orbifolds, and their fate under closed string tachyon condensation. We expect tools from toric geometry, associated with the D-brane probe theory to be useful in this study. Namely, using toric geometry techniques, one can hope to understand the behaviour of higher dimensional non-supersymmetric orbifolds (for which a canonical resolution is not available) under tachyonic decay. A related issue that one might address is whether this decay of space-time is more generic. Namely, given a generic background which breaks space-time supersymmetry, is there a process in string theory itself, which would lead to a decay of this background into a supersymmetric string background.

It is these issues that we address in this paper. As a first step, we partially generalise the D-brane probe results of APS for non-supersymmetric orbifolds of the form $\mathbb{C}^{2} / \Gamma$. From the probe point of view, the D-brane can see only certain types of decays, and we address the question of the classification of such theories that flow in the IR to supersymmetric orbifolds. Next, we study the chiral ring techniques of HKMM as applied to non-supersymmetric orbifolds of the form $\mathbb{C}^{2} / \Gamma$, which are related to the issue of these decays seen from the point of view of toric geometry in two complex dimensions. Our interest is two-fold. Firstly, a toric geometry picture of the decay process for two-fold orbifolds would be an useful tool in understanding such processes in higher dimensions. Secondly, one would, 
via this picture, be able to study, for example, the decay of generic weighted projective spaces using the picturisation of D-branes in weighted projective spaces as D-branes on the resolutions of (higher-dimensional) orbifolds [11. This study has already been initiated in [5], and our methods are complimentary to the mirror symmetry principles used there. Further, we study the inverse toric procedure pioneered in [17] for some simple examples. This procedure is expected to play an important role in the full understanding of the decay of non-supersymmetric backgrounds, as we will point out in the paper.

This paper is organised as follows. In section 2, we briefly review the probe brane analysis of APS, and study some flow patterns for the $\mathbb{C}^{2}$ orbifold. Section 3 begins with a brief review of the chiral ring analysis of HKMM, after which we discuss the toric geometry of the D-branes probing non-supersymmetric orbifolds, and discuss several examples, both for two and three-fold orbifolds. Next, we study the inverse toric procedure, applied to non-supersymmetric orbifolds and initiate a discussion on toric duality for non-supersymmetric orbifolds. Section 4 ends with some discussions and conclusions.

\section{D-brane Probes of Non-Supersymmetric Orb- ifolds}

In [2], APS has studied closed string theory on non-supersymmetric orbifold backgrounds. As we have mentioned before, these break space-time supersymmetry, and have tachyonic modes in (some of) the twisted sectors. An important aspect of the theories that have been studied is that the tachyonic excitations are localised at the fixed points of the orbifolds, and do not affect the stability of the bulk space-time. It was shown in [2] that these orbifolds decay with time, and the final theory reached via this decay process is a supersymmetric orbifold. There are two distinct scales involved in this problem. First, one can study the decay process in the sub-stringy regime, where the tachyon expectation value is small. Here, one expects the world volume gauge theory of D-branes probing these orbifolds to provide an useful tool in studying the decay process. In the substringy regime, one can study the world volume gauge theory of a D-brane that probes the non-superymmetric orbifold, using quiver diagram techniques developed in [8]. (Here, one is dealing with the classical worlvolume gauge theory that lives on the branes). Far from the substringy regime, when $\alpha^{\prime}$ corrections 
become large, the probe analysis is not suitable any more, and one has to revert to a gravity analysis.

These two approaches were studied in [2] for the case of orbifolds of the form $\mathbb{C} / \mathbb{Z}_{n}$. It was shown that these non-supersymmetric orbifolds decay to orbifolds of lower rank, and the process continues until one reaches flat space. Similarly, for $\mathbb{C}^{2} / \Gamma$ orbifolds, brane probes lead to the prediction of transitions from nonsupersymmetric orbifolds to supersymmetric orbifolds (of lower rank). The probe analysis is again done for the sub-stringy regime. The method of [2] is to excite marginal deformations in the original theory, which takes the system to a lower rank orbifold, which is only locally supersymmetric. Deformations of this latter theory, which are expected to be tachyonic in nature, then drives the system to a supersymmetric configuration.

It is important to turn on only marginal perturbations in this method of probing a non-supersymmetric orbifold. If one turns on generic tachyonic deformations, quantum corrections will become important, and the the classical brane probe theory ceases to be useful. In exciting marginal deformations in the theory, one has to maintain a certain quantum symmetry out of the full symmetry group. This quantum symmetry is retained by the D-brane theory once one breaks the other part of the orbifold group. We will now elaborate on this in some more details.

\subsection{General Pattern for Quivers}

Let us begin by considering D-brane probes of Type II string theory on the $\mathbb{C}^{2}$ orbifold based on the general twist

$$
R=\exp \left\{\frac{2 \pi i}{n}\left(J_{67}+k J_{89}\right)\right\}
$$

where $J_{67}$ and $J_{89}$ refer to the rotations in the complex planes $Z^{1}=X^{6}+i X^{7}$ and $Z^{2}=X^{8}+i X^{9}$. We can consider a D-p brane probe of the above geometry, where the brane extends only along the transverse directions. The low energy theory of such a configuration is the orbifold of the $N=4$ world volume gauge theory on the D-brane, with the usual [8] projection conditions. Following the notation of [2], we call this orbifold $\mathbb{C}^{2} / \mathbb{Z}_{n(k)}$. The world volume spinors in the 16 of $S O(9,1), \eta$ and $\chi$, are labelled by their weights under $S O(4)$, and the $S O(5,1)$ spinor indices are suppressed [2]. In this notation, $\eta$ is the $(-,+)$ component, and $\chi$ is the $(-,-)$ component. 
The quiver diagram is obtained by following the by now standard prescription due to Douglas and Moore $[\mathbb{8}]$, and will have $n$ nodes, corresponding to the $n U(1)$ factors of the gauge group $U(1)^{n}$. As we have mentioned, an orbifold of this form will not preserve space-time supersymmetry, and will flow (in the sense of the RG) to a supersymmetric orbifold via the condensation of twisted sector tachyons. It is an interesting question to classify these flows using general quiver techniques, and we will present some results on the decay of non-supersymmetric orbifolds by turning on marginal deformations. A complete classification of generic flows using the methods of [2] is a difficult issue to address. For the purpose of this paper, we will make the simplifying assumption of turning on only marginal perturbations, but, as we will see later in the paper by using tools from toric geometry, there are several interesting aspects of such flows.

Let us begin by reviewing the procedure due to APS for the decay of a nonsupersymmetric orbifold singularity. As we have already mentioned, the essential idea is to turn on marginal or tachyonic deformations from a given twisted sector, which is expected to produce a partial blowup of the initial singularity. Once the system has reached such a stage, one can consider turning on further deformations which are tachyonic in nature, and drives the system to a supersymmetric configuration.

In terms of the analogues of the $\mathrm{F}$ and $\mathrm{D}$ terms that appear in the D-brane world volume gauge theory in the supersymmetric case, this is tantamount to turning on certain Fayet-Illiapoulos (FI) parameters, in a way that a quantum symmetry is maintained at the end. After choosing a particular vaccum, in which we gives vev's to a certain set of fields maintaining this symmetry, we are left with a reduced world volume theory (integrating out fields that become massive due to the vev's) that, upon suitable rearrangement, can be seen to correspond to a different (lower rank) orbifold action.

In particular, from eq.(何), one can reach a configuration that preserves a $\mathbb{Z}_{m}$ symmetry, where $m$ divides $n$. This might be achieved by turning on marginal deformations from an appropriate twisted sector.

There are two distinct choices of vaccum corresponding to turning on vevs for the fields that parametrise either of the two $\mathbb{C}^{2}$ directions. In either case, the final symmetry group that is restored will depend on the symmetry of terms that take vev's. It turns out that the analysis of the vacua that preserves an $m$ fold symmetry coming from the vevs of the $Z^{1}$ is qualitatively different from the corresponding vacua where one chooses the $Z^{2}$ vevs. Let us now study this in 
some details.

\subsection{Reaching a supersymmetric configuration}

In what follows, we will study a class of non-supersymmetric orbifolds of the form $\mathbb{C}^{2} / \mathbb{Z}_{n(k)}$ that flow to orbifolds of the form $\mathbb{C}^{2} / \mathbb{Z}_{m\left(k^{\prime}\right)}$ where $m$ is a factor of $n$. Flows starting from the non-supersymmetric orbifold of the form $\mathbb{C}^{2} / \mathbb{Z}_{2 l(2 l-1)}$ and $\mathbb{C}^{2} / \mathbb{Z}_{2 l(3)}$ have been considered in [2] (with $m$ being 2 and $l$ respectively). In general, the final supersymmetric configuration will be of the form $\mathbb{C}^{2} / \mathbb{Z}_{m(1)}$ [2], and will have the interpretation of having an opposite supersymmetry from the usual $\mathbb{C}^{2} / \mathbb{Z}_{m}$ orbifold. How does a D-brane see such a decay ? Let us take an example. Consider the orbifold $\mathbb{C}^{2} / \mathbb{Z}_{8(3)}$. The quotienting group $\mathbb{Z}_{8}$ has the subgroups $\mathbb{Z}_{2}$ and $\mathbb{Z}_{4}$. Taking the second or the fourth power of $R$ in eq. (1), we obtain

$$
R^{2(4)}=\exp \left\{\frac{2 \pi i}{4(2)}\left(J_{67}+3 J_{89}\right)\right\}
$$

Taking the fermionic part of the string theory into account, $\mathbb{C}^{2} / \mathbb{Z}_{2(3)}$ (or equivalently $\mathbb{C}^{2} / \mathbb{Z}_{2(-1)}$ is a supersymmetric background, whereas $\mathbb{C}^{2} / \mathbb{Z}_{4(3)}$ is not. Hence, only a deformation by $R^{4}$ will be marginal. If we turn on the marginal deformation corresponding to the fourth subsector of the theory, we get, as the end product, the orbifold $\mathbb{C}^{2} / \mathbb{Z}_{4(1)}$ [2].

The procedure of APS is to generate vevs for the fields of the theory in such a way as to maintain a certain subgroup of the initial orbifold group, corresponding to the turning on of an appropriate twisted sector. The vev breaks the other part of the group action, and we are left finally with the subgroup that we had maintained in choosing the vevs. Of course, one might expect that such a constraint is not necessary. Namely, by choosing arbitrary vevs for certain fields in the Dbrane gauge theory, one might still reach a supersymmetric configuration. Such an example, analysed in [2] is the decay of the orbifold $\mathbb{C}^{2} / \mathbb{Z}_{5(2)}$ to a supersym-

metric configuration. Here, the deformations are entirely tachyonic, there being no marginal deformations in any of the twisted sectors. As we have pointed out, it is difficult to classify completely such generic deformations using the methods of this subsection, and we will not treat this issue here.

Let us concentrate on the cases where the quotienting group admits of discrete subgroups, i.e, the cases $\mathbb{C}^{2} / \mathbb{Z}_{n(k)}$ where $n$ admits of factors greater than unity. By turning on marginal perturbations that corresponds to maintaining a 
discrete subgroup of the initial quotienting group, we can reach supersymmetric configurations. This restricted class of flows can be classified by noting that since the final configuration is guaranteed to be of the form $\mathbb{C}^{2} / \mathbb{Z}_{n^{\prime}(1)}$, we expect loops in the final (annealed) quiver diagram, arising out of the spinors $\eta$ or $\bar{\eta}$, as can be seen by inspecting their spin. Therefore, we need to choose the vaccum of the original theory in such a way that these loops are produced in the final quiver diagram. In general, if there are several supersymmetric sectors in a non-supersymmetric orbifold, the final supersymmetric configuration might be reached in several steps, we will return to this question in a while.

\subsection{Turning on VEVs for the $Z^{1}$ and $Z^{2}$}

For the orbifold action of eq. (11), the surviving components of the coordinates $Z^{1}\left(X^{6}+i X^{7}\right)$ after projection by the orbifolding group takes the form $Z_{i j}^{1}$ where

$$
(i, j):(1,2),(2,3), \cdots(n-1, n)
$$

Now, we wish to turn on marginal deformations so that the symmetry group $\mathbb{Z}_{m}$ is maintained. This would involve identifying the fields under an $m$ fold symmetry and choosing a vaccum that restores this symmetry. For example, one set of the fields $Z_{i j}^{1}$ that are identified, are,

$$
(i j):(1,2),\left(1+\frac{n}{m}, 2+\frac{n}{m}\right), \cdots\left(1+(m-1) \frac{n}{m}, 2+(m-1) \frac{n}{m}\right)
$$

and similar identifications hold for other sets of fields. In order to maintain an $m$ fold symmetry in the final orbifold theory, one choice of the massless fields is given by $Z_{\frac{n}{m} j, \frac{n}{m} j+1}^{1}$, with the rest of the $Z^{1}$ components acquiring vevs. Now, in order to determine the massless fermions, we need to inspect the Yukawa terms, which are of the form $L_{Y}=\operatorname{Tr}\left\{\left[Z^{1}, \chi\right] \eta+\left[Z^{2}, \chi\right] \bar{\eta}+h . c\right\}$ [8], [2]. It suffices to consider the first term in this case, and with our choice of the massless components of $Z^{1}$, the massless components of $\eta$ are determined from the term

$$
\begin{aligned}
& {\left[Z_{\frac{n}{m} j, \frac{n}{m} j+1}^{1} \chi_{\frac{n}{m} j+1, \frac{n}{m} j+\frac{2 n-k+1}{2}}-\chi_{\frac{n}{m} j, \frac{n}{m} j+\frac{2 n-k-1}{2}} Z_{\frac{n}{m} j+\frac{2 n-k-1}{2}, \frac{n}{m} j+\frac{2 n-k+1}{2}}^{1}\right]} \\
& \times\left(\eta_{\frac{n}{m} j} j+\frac{2 n-k+1}{2}, \frac{n}{m} j\right)
\end{aligned}
$$

Here $j$ is an integer, which, without loss of generality, we can choose to be unity. First, notice that in order to get a sensible final quiver where the massless $\eta$ 's arise as loops, we require the remaining fermionic fields (of the final fermion quiver) 
to arise as massless linear combinations of the $\chi$ 's. (In general, such massless combinations will appear with the massive components of $Z^{1}$ ). For these to occur, both the components of $Z^{1}$ appearing in (5) need to be massless, and we see from index matching that such massless combinations will occur only when

$$
(k+1)=2 p \frac{n}{m}
$$

where $p \in \mathbb{Z}$. This condition is actually the same as that for the existance of supersymmetric subsectors in the sector twisted by $m$, as can be seen by taking the $m$ th power of $R$ in eq. (1). To obtain loops for the $\eta$ 's in the final quiver, we see by inspecting the indices of the $\eta$ fields in eq. (5), that the following constraint has to be satisfied

$$
\frac{n}{m}+\frac{1}{2}(1-k)=b(\bmod n)
$$

where $b=1,2, \cdots \frac{n}{m}$. (We can see this by setting $j=1$ in (5), and noting that the bosonic vevs identify the nodes $1,2, \cdots \frac{n}{m}$ in the original quiver diagram). Combining (7) and (6), we obtain the condition

$$
(1-p) \frac{n}{m}+1=b(\bmod n)
$$

Which is satisfied by $p=1$, which, as we will see, will be the case for most of our examples.

From the above discussion, it follows that the following flow is possible by turning on the $Z^{1}$ vevs

$$
\mathbb{C}^{2} / \mathbb{Z}_{j l(2 j-1)} \rightarrow \mathbb{C}^{2} / \mathbb{Z}_{l(1)}
$$

here, $p=1$ from eq. (6), and eq. (7) is satisfied. As a special case of this equation, we see the flow patterns from the above equation

$$
\begin{aligned}
\mathbb{C}^{2} / \mathbb{Z}_{2 l(3)} & \rightarrow \mathbb{C}^{2} / \mathbb{Z}_{l(1)} \\
\mathbb{C}^{2} / \mathbb{Z}_{2 l(2 l-1)} & \rightarrow \mathbb{C}^{2} / \mathbb{Z}_{2(1)}
\end{aligned}
$$

which have been considered in [2].

Let us now consider the example of a non-supersymmetric orbifold that has more than one supersymmetric subsector. Consider, for example, the flow

$$
\mathbb{C}^{2} / \mathbb{Z}_{12(7)} \rightarrow \mathbb{C}^{2} / \mathbb{Z}_{3(1)}
$$

This satisfies (6) with $p=1$, and corresponds to turning on marginal deformations from the third twisted sector. However, one could consider turning on 
marignal deformations from the sixth twisted sector, which is also supersymmetric. Marginal deformations from this sector, however, does not make the final orbifold supersymmetric, because, for a final $\mathbb{Z}_{6}$ symmetry, we find that equation (7) is not satisfied with $n=12$ and $m=6$ ( $p=2$ in this example). It can be checked that this flow is

$$
\mathbb{C}^{2} / \mathbb{Z}_{12(7)} \rightarrow \mathbb{C}^{2} / \mathbb{Z}_{6(3)}
$$

The orbifold on the r.h.s will further decay into the supersymmetric orbifold, $\mathbb{C}^{2} / \mathbb{Z}_{3(1)}$. This is an example of a double-decay process. Some other examples of such decays, by turning on vevs for the $Z^{1}$ are

$$
\begin{aligned}
& \mathbb{C}^{2} / \mathbb{Z}_{12(11)} \rightarrow \mathbb{C}^{2} / \mathbb{Z}_{4(3)} \rightarrow \mathbb{C}^{2} / \mathbb{Z}_{2(1)} \\
& \mathbb{C}^{2} / \mathbb{Z}_{12(11)} \rightarrow \mathbb{C}^{2} / \mathbb{Z}_{6(5)} \rightarrow \mathbb{C}^{2} / \mathbb{Z}_{2(1)}
\end{aligned}
$$

Here, from the initial orbifold, by exciting the fourth and the sixth twisted sectors (both of which have marginal deformations), one can reach an identical configuration, via two different routes. The last stage of the decay, in both the cases, satisfies the condition in eq. (7) with $p=1$.

To summarize the discussion so far, we have seen that for orbifolds which have multiple supersymmetric twisted sectors (in the sense of [2]), a marginal deformation that drives the orbifold into a supersymmetric one must obey the condition in eq. (7). If we excite a marginal deformation that does not satisfy these equations, the orbifold flows into a non supersymmetric orbifold of lower rank, from which it finally decays, by marginal deformations, into a supersymmetric orbifold, and in the final step, the conditions in eq. (7) is satisfied.

Let us now consider another class of examples where an $l$ fold final symmetry is preserved starting from a $2 l$ fold symmetry, but for which eq. (9) is not satisfied. For eg. consider the orbifold $\mathbb{C}^{2} / \mathbb{Z}_{2 l(2 l+1)}$ for odd $l$. In this case, after effecting the orbifold projection in the D-brane gauge theory, the surviving components of the $Z^{1}$ and $Z^{2}$ are both of the form $Z_{j, j+1}^{i}$ for $j=1,2, \cdots 2 l$. The components of the $S O(9,1)$ spinors which survive, are $\chi_{j, j+l-1}^{(--)}$and $\eta_{j, j+l}^{(-+)}$. If we now give vevs to $Z_{2 j-1,2 j}^{1}$, the bosonic fields that remain massless, are $Z_{2 j, 2 j+1}^{1}$ and $Z_{2 j-1,2 j}^{2}$. Similarly, the fermion components that remain massless can be shown to be $\eta_{2 j-1,2 j-1+l}$ and the linear combinations of the $\chi$ fields, namely $\left(\chi_{2 j-1,2 j+l-2}+\chi_{j 2,2 j+l-1}\right)$. With these, the final orbifold is seen to be non-supersymmetric, and of the form form $\mathbb{C} / \mathbb{Z}_{l} \times \mathbb{C}$. This will finally decay into flat space via further twisted sector tachyon condensation as discussed in [2]. In 


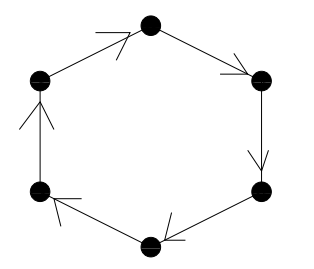

(a)



(b)

(c)

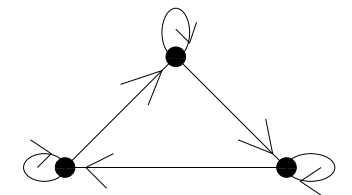

(d)

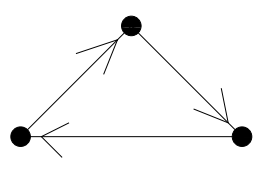

(e)

Figure 1: Quiver Diagram for the process $\mathbb{C}^{2} / \mathbb{Z}_{6(7)} \rightarrow \mathbb{C} / \mathbb{Z}_{3(1)} \times C$. (a) shows the initial bosonic quivers for the two worldvolume scalars, (b) and (c) are the quivers for the $S O(9,1)$ fermions. (d) shows the final quiver for the bosons and (e) shows the final quiver for both the fermionic fields.

summary, the flow pattern just discussed is

$$
\mathbb{C}^{2} / \mathbb{Z}_{2 l(2 l+1)} \rightarrow \mathbb{C} / \mathbb{Z}_{l} \times \mathbb{C}
$$

The initial and final quiver diagrams for one such process, $\mathbb{C}^{2} / \mathbb{Z}_{6(7)} \rightarrow \mathbb{C} / \mathbb{Z}_{3(1)} \times C$ is shown in figure (11).

A similar analysis can be performed to study the flow of non-supersymmetric orbifolds upon turning on the vevs of the $Z^{2}$ fields. As before, we can study the index structure of the surviving fields in order to determine the flow patterns. As has already been noted in [2], turning on the $Z^{2}$ vevs will, in general, drive the initial orbifold to a configuration different from the one reached with corresponding $Z^{1}$ vevs. For example, the non-supersymmetric orbifold $\mathbb{C}^{2} / \mathbb{Z}_{2 l(3)}$ has the following flow pattern

$$
\mathbb{C}^{2} / \mathbb{Z}_{2 l(3)} \rightarrow \mathbb{C}^{2} / \mathbb{Z}_{l(1)} \oplus \mathbb{C}^{2} / \mathbb{Z}_{l(-3)}
$$

The two terms on the r.h.s of the above equation are obtained by marginal deformations from the $l$ th twisted sector by turning on vevs for $Z^{1}$ and $Z^{2}$ respectively. Whereas the flow effected by the $Z^{1}$ vev has, as the endpoint, a supersymmetric orbifold, that by the $Z^{2}$ vev is non-supersymmetric for $l>2$ (for a possibility of such transitions for higher values of $l$, see [6]). In general, for non-supersymmetric orbifolds of the form $\mathbb{C}^{2} / \mathbb{Z}_{n(l)}$, the analysis of the index structure of the surviving 
fields have to be done case by case. The results, we believe, are not very illuminating, considering the fact that such analysis as presented in this subsection can be done with relative ease only for cases where marginal deformations are turned on, thus restricting its applicability. We will therefore proceed to study the Dbrane gauge theory on non-supersymmetric orbifolds, using chiral ring methods and toric geometry. However, before ending this section, let us point out that a similar quiver diagram analysis can be done for D-branes probing orbifolds of the form $\mathbb{C}^{3} / \mathbb{Z}_{n(k, m)}$ where $k$ and $m$ are integers that arise in the orbifold action

$$
R=\exp \frac{2 \pi i}{n}\left(J_{45}+k J_{67}+m J_{89}\right)
$$

As in the two-fold example, constrains on $k$ and $m$ arise due to conditions of localisation of the tachyon. We will briefly mention this class of examples in the next section.

\section{Chiral Ring Techniques and Toric Geometry Methods}

In the NS-R formalism, the string world sheet conformal field theory has $\mathcal{N}=2$ supersymmetry, and is endowed with ring of (anti)chiral primary operators in the NS sector. By extending the 1-1 correspondence between the chiral ring and the geometric blow-up modes to relevant perturbations [6], the decay of nonsupersymmetric orbifolds can be studied from a geometric point of view.

To summarize the construction of the chiral ring [12] [6], we recall that for theories on orbifolds of the form $\mathbb{C}^{d} / \mathbb{Z}_{n}$ there is one twisted sector associated with each element $g^{j} \in \mathbb{Z}_{n}, j=1,2, . ., n-1, g^{n}=1$. In each twisted sector (for each complex dimension which is orbifolded), there is a bosonic and a fermionic twist operator that can be combined to form the building blocks for the chiral operators of the worldsheet theory. Bosonizing the fermionic fields as $\psi_{i}=e^{H_{i}}$, the twisted sector chiral operators can be written in the case of two-fold orbifolds of the form $\mathbb{C}^{2} / \mathbb{Z}_{n(k)}$ as 6

$$
X_{j}=X_{j}^{(1)} X_{n\left\{\frac{j k}{n}\right\}}^{(2)}, \quad X_{j}^{(1)}=\sigma_{\frac{j}{n}} \exp \left[i\left(\frac{j}{n}\right)\left(H_{1}-\bar{H}_{1}\right)\right]
$$

where (1) and (2) denote the two complex directions that are orbifoldized, $\sigma_{\frac{j}{n}}$ is the bosonic twist $j$ operator [12], and in the above equation, a similar expression 
holds for the operator $X^{(2)}$ as for $X^{(1)}$. As usual, $j=1, \cdots n-1$ label the twisted sectors, and $\{x\}$ denotes the fractional part of $x$. The R-charges (with respect to the world sheet $U(1)$ current) of these operators are given by

$$
R_{j}=\frac{j}{n}+\left\{\frac{j k}{n}\right\}
$$

Now, the chiral GSO projection for Type II strings acts on the bosonised fermions as

$$
H_{1} \rightarrow H_{1}-k \pi ; \quad H_{2} \rightarrow H_{2}+\pi
$$

Considering the fact that in the untwisted sector of the orbifold, this must reduce to the standard $(-1)^{F_{L}}, k$ is fixed to be odd as in the D-brane probe analysis of [2]. Marginal deformations of the CFT correspond to perturbations by operators which have $R_{j}=1$ [13], and there is an 1-1 correspondence of these with the blow-up modes of the orbifold. This correspondence can be extended [6] to the relevant modes $\left(R_{j}<1\right)$ which correspond to tachyonic excitations in space-time, by using a toric geometry description for the singularity. However the R-charge being non-integral, the spectral flow argument for the correspondence does not hold here.

Several flow patterns have been analysed in [6], and the $g_{c l}$ conjecture has been verified for these. In the brane probe analysis of the last section, we restricted ourselves to considering only marginal deformations of the D-brane theory. Whenever this arises as special cases in the analysis of HKMM, the results are seen to agree.

The above formalism can be generalised to the case of 3-fold orbifolds, where the action of discrete group is as in (16). For the case of 3-fold orbifolds, however, a canonical resolution does not exist, unlike the two-fold examples. We will consider the simplest class of examples for these three-folds in a while, in which we set, in eq. (16), $k=1$. These non-supersymmetric three-fold orbifolds will, in general, have tachyonic excitations, and, following [2] or [6], the condition for the localisation of tachyons in these cases can be shown to imply that the integer $m$ in (16) is even (with $k=1$ ). As in [6], one can again construct the chiral ring and analyse the structure of flows. We will, however, perform an equivalent analysis for these cases in terms of the toric geometry of the probe branes in the next subsection. 


\subsection{Using Tools From Toric Geometry}

We now explore the above processes exemplifying the decay of space-time by using methods of toric geometry. As we have already mentioned, toric geometry is an extremely useful tool in studying singular spaces. However, as a caveat, we note that the method, which we will now elaborate, concerns only the bosonic subsector of the string theory. This is not a problem however, since the corresponding fermions can be introduced at any stage of the calculation. The methods that we will use are fairly standard in the mathematics literature [14], [18]. For the relevant physics, the reader is referred to [15], [16] and references therein.

We will deal with orbifolds of the form $\mathbb{C}^{m} / \Gamma$, where $\Gamma$ is the discrete group $\mathbb{Z}_{n}$. For the moment, we concentrate on the case $m=2$, where the action on the complex coordinates of the discrete group on $\mathbb{C}^{2}$ is given by

$$
\left(Z_{1}, Z_{2}\right) \rightarrow\left(\omega Z_{1}, \omega^{k} Z_{2}\right)
$$

where $\omega=\exp [2 \pi i / n]$ is the $n$th root of unity, and $k$ is an integer, with $|k|<n$. This is the Hirzebruch-Jung singularity, and following the notation of APS, this singularity is denoted by $\mathbb{C}^{2} / \mathbb{Z}_{n(k)}$.

The toric variety for the correspoding non-singular (i.e resolved) geometry is given by specifying a set of lattice points in the two dimensional lattice (with $S L(2, \mathbb{Z})$ automorphism) generated by the unit vectors $(1,0)$ and $(0,1)$, which we call $e_{1}$ and $e_{2}$. Given a singularity of the form $\mathbb{C}^{2} / \mathbb{Z}_{n(k)}$, the toric diagram of its minimal resolution is given by the cone generated by two vectors, $v_{f}=e_{2}$ and $v_{i}=\left(n e_{1}-k e_{2}\right)$. It can be shown [14], [18] that there are $r$ added vertices inside the cone (corresponding to the blow up modes) generated by $v_{f}$ and $v_{i}$, and these are determined from the relations

$$
a_{i} v_{i}=v_{i-1}+v_{i+1}
$$

where the coefficients $a_{i} \geq 2$ and are the integers appearing in the HirzebruchJung continued fraction

$$
\frac{n}{k}=a_{1}-\frac{1}{a_{2}-\frac{1}{\cdots-\frac{1}{a_{r}}}}
$$

where it is understood that for $k \equiv n+k$ for $k<0$. Each interior vector is an exceptional divisor, and correspond to the blowing up of $\mathbb{P}^{1} \mathrm{~s}$, with self intersection number $-a_{i}$. We will follow the standard notation, where the continued fraction is denoted by $\left[a_{1}, a_{2}, \cdots, a_{r}\right]$. 
Let us take the example of the supersymmetric orbifold $\mathbb{C}^{2} / \mathbb{Z}_{4(-1)}$, considered in [6]. The corresponding (non-singular) toric variety is generated by the set of five vectors, which are, $(0,1),(1,0),(2,-1),(3,-2),(4,-3)$. Note that the toric data of this orbifold is the same as that for $\mathbb{C}^{2} / \mathbb{Z}_{4(3)}$. The latter is not spacetime supersymmetric. We will keep this in mind, it being implied that whether the orbifold is supersymmetric or not can be checked by taking into account the fermionic quiver diagram. The toric data can be arranged in an array

$$
\mathcal{T}=\left(\begin{array}{ccccc}
1 & 0 & -1 & -2 & -3 \\
0 & 1 & 2 & 3 & 4
\end{array}\right)
$$

The continued fraction for this example is $[2,2,2]$. In [6], it was shown that perturbing the Lagrangian of the closed string theory probing this orbifold by a chiral primary operator, and taking the coupling of this operator to be very large, corresponds to a splitting up of the space. Noting that the perturbation corresponds to the blowing up of an appropriate $\mathbb{C P}^{1}$, and the operation of taking the coupling to infinity is to effectively blow up this $\mathbb{C P}^{1}$ to infinite size (and hence to decouple it from the geometry), the splitting is denoted by

$$
\mathbb{C}^{2} / \mathbb{Z}_{n(-1)} \rightarrow \mathbb{C}^{2} / \mathbb{Z}_{j(-1)} \oplus \mathbb{C}^{2} / \mathbb{Z}_{n-j(-1)}
$$

It is easy to understand this process from toric diagrams. Splitting up the toric cone will correspond to a split of the toric data into two parts, using any one interior vector twice. For example, the data in (23) can be split into

$$
\left(\begin{array}{ccc}
1 & 0 & -1 \\
0 & 1 & 2
\end{array}\right) \text { and }\left(\begin{array}{ccc}
-1 & -2 & -3 \\
2 & 3 & 4
\end{array}\right)
$$

The first matrix can be recognised to be the toric data of the resolution of the orbifold $\mathbb{C}^{2} / \mathbb{Z}_{2(1)}$. Using the automorphism of the two dimensional lattice, the second matrix, after a transformation by the $S L(2, Z)$ matrix $\left(\begin{array}{cc}3 & 2 \\ -2 & -1\end{array}\right)$, can also be brought into the form $\left(\begin{array}{ccc}1 & 0 & -1 \\ 0 & 1 & 2\end{array}\right)$. This is the analogue of the flow pattern of (24), namely,

$$
\mathbb{C}^{2} / \mathbb{Z}_{4(-1)} \rightarrow \mathbb{C}^{2} / \mathbb{Z}_{2(-1)} \oplus \mathbb{C}^{2} / \mathbb{Z}_{2(-1)}
$$

Let us point out at this stage that deforming the CFT by marginal operators will, in general, correspond to splitting the toric data along a vector that lies on the edge of the toric cone connecting $v_{i}$ and $v_{f}$. We will come back to this later. 
Let us now take another example. Consider, for eg., the orbifold $\mathbb{C}^{2} / \mathbb{Z}_{10(-3)}$, which is non-supersymmetric. The toric data for this orbifold is given by

$$
\mathcal{T}=\left(\begin{array}{ccccc}
1 & 0 & -1 & -2 & -7 \\
0 & 1 & 2 & 3 & 10
\end{array}\right)
$$

corresponding to the continued fraction $[2,2,4]$. As shown in [6], deforming by the generators of the closed string CFT corresponding to this orbifold produces the flow

$$
\mathbb{C}^{2} / \mathbb{Z}_{10(-3)} \rightarrow \mathbb{C}^{2} / \mathbb{Z}_{2(-1)} \oplus \mathbb{C}^{2} / \mathbb{Z}_{4(-3)}
$$

It is simple to see this from (27), just by splitting the data into two parts, with the common vector being $(2,-1)$ and using an appropriate $S L(2, Z)$ transformation. In this example, there is a second way to split the data. Note that the blowing up of the point of intersection of the $j$ th and the $j+1$ st $\mathbb{C P}^{1}$ is described, in the toric language, by the following change in the corresponding continued fraction [14]

$$
\left[a_{1}, a_{2}, \cdots, a_{r}\right] \rightarrow\left[a_{1}, a_{2}, \cdots,\left(a_{j}+1\right), 1,\left(a_{j}+1\right), \cdots, a_{r}\right]
$$

This corresponds to inserting a vector $v$ between $v_{j}$ and $v_{j+1}$ such that $v=$ $v_{j}+v_{j+1}$. One way to do this is to modify the toric data to the following

$$
\mathcal{T}=\left(\begin{array}{ccccc}
1 & 0 & -1 & -2 & -7 \\
0 & 1 & 2 & 3 & 10
\end{array}\right) \equiv\left(\begin{array}{cccccc}
1 & 0 & -1 & -3 & -2 & -7 \\
0 & 1 & 2 & 5 & 3 & 10
\end{array}\right)
$$

The self intersection numbers from the above data is seen to be $[2,3,1,5]$ according to (29). Now, in an obvious way, we can split the toric data into two parts, which can be recognised as corresponding to $\mathbb{C}^{2} / \mathbb{Z}_{5(3)}$ and $\mathbb{C}^{2} / \mathbb{Z}_{5(1)}$.

In general, there will be more than one way to effect the above splitting, corresponding to the number of ways in which one can change the continued fraction, as in (29). These are related to perturbations of the CFT by products of generators of the chiral ring [6].

At this point, let us mention that the same analysis can be done for toric singularities of the form $\mathbb{C}^{3} / \Gamma$. Consider the singularities of the form (16), where for simplicity, we set $k=1$. We will refer to this class of singularities as $\left[\frac{1}{n}(1,1, m)\right]$. These orbifolds are generally non-supersymmetric, and for these cases, localisation of the tachyons require that $m$ is even. A few of the non-supersymmetric cases will be treated in the next subsection. Here, we will briefly discuss the simplest supersymmetric case, $\left[\frac{1}{3}(1,1,1)\right]$ [3], [19]. (As is well known, when $n$ is not a prime number, these orbifold will have non-isolated singularities. These, in 
particular, include the cases when $n$ is even. We will come back to this class of singularities in a while). Recall [3], [18] that for the three-fold quotient singularity of the form $\left[\frac{1}{n}(1, k, m)\right]$, the toric fan is given by a cone in a three-dimensional lattice, generated by the following one dimensional cones

$$
v_{1}=\left(n e^{1}-k e^{2}-m e^{3}\right), \quad v_{2}=e^{2}, \quad v_{3}=e^{3}
$$

where $\left(e^{1}, e^{2}, e^{3}\right)$ are the three unit vectors that form a basis for the three dimensional lattice. The blowup of the singularity is effected by adding points in the toric diagram which are linear combinations of the above vectors, with weights determined by the action of the discrete group (for details, the reader is referred to [3]). These internal vectors are of the form

$$
u_{j}=\sum_{i=1}^{3} t_{i} v_{i}, \quad 0 \leq t_{i} \leq 1
$$

For our example $\left[\frac{1}{3}(1,1,1)\right]$, The one dimensional cones are given by

$$
v_{1}=(3,-1,-1), \quad v_{2}=(0,1,0), \quad v_{3}=(0,0,1)
$$

In this case, we can add one internal ray as in (32), with weights $t_{i}=\frac{1}{3}, i=$ $1,2,3$, which is given by the vector $(1,0,0)$. This is a blowup of the singularity $\left[\frac{1}{3}(1,1,1)\right]$.

It is simple to generalise the procedure of [6] in studying this class of examples. Namely, we can study marginal perturbations, which correspond to perturbations by the generators of the chiral ring in these examples, and, sending the coefficients of these marginal modes to infinity, we flow to orbifolds of lower rank. We can see it directly from the toric data, using the $S L(3, Z)$ automorphism of the three dimensional lattice, much like the two-fold examples. In the particular case of the orbifold $\left[\frac{1}{3}(1,1,1)\right]$, using the added vector $(1,0,0)$, it is easy to see, in analogy with the two-fold examples, that the toric data can be split up into that for three simpler spaces, all of which can be identified with the flat space $\mathbb{C}^{3}$. Similar analyses can be carried out for higher rank supersymmetric orbifolds, and in a method similar to the two-fold examples, we have checked from the toric data for these orbifolds, that they flow to lower rank supersymmetric orbifolds.

We now move on to the D-brane gauge theory descrpition of the singularities that we have considered. 


\subsection{D-brane Gauge Theory}

In the supersymmetric case, the toric data corresponding to a certain quotient singularity can be succinctly described in terms of the gauge theory living on the D-brane probing the singularity. The gauge theory is constructed by following the well known prescription of Douglas and Moore [8]. Such a gauge theory, in general, is described by its matter content and its interactions. While the former is specified by the D-term equations, the latter is given by the superpotential, which lead to the F-term constraints. The data can be combined in a form prescribed in [19] in order to extract geometric information of the singularity that the D-brane probes.

An analogous procedure can be followed for the non-supersymmetric orbifolds that we have been considering, purely by considerations of the bosonic subsector of the theory in the classical limit. The analogy is, in a sense, clear. When the tachyon expectation values are small compared to the string scale, i.e in the substringy regime, we can set up an analogue of the prescription of [19, by examining the classical moduli space of the scalar fields. Of course, when generic twisted sectors are turned on, the probe analysis will be less useful. But for the moment, let us assume that we are working in a regime where fluctuations are very small, and one can use the classical picture.

Our analysis is similar to the supersymmetric case. Let us illustrate this by an example which will also serve to set up the notations that we will use later. Consider the non-supersymmetric orbifold $\mathbb{C}^{2} / \mathbb{Z}_{5(3)}$. This orbifold does not have any supersymmetric subsectors, and hence any deformation of the theory will be purely tachyonic. In this case, the low energy theory will be an orbifold of the D-brane gauge theory in flat space, obtained in the usual way [8] by the action of the discrete group on the coordinates and the Chan-Paton indices. By considering the classical scalar potential, one can write down the equivalents of the $\mathrm{D}$ and $\mathrm{F}$ terms.

The projection of the fields is as in [8], and the quiver diagram can be obtained by standard techniques. This quiver diagram encodes information about the $U(1)^{5}$ charges of the unprojected fields. This can be written as a matrix $\Delta$, where one of the overall $U(1)$ s denoting the centre of mass motion of the branes, 
is omitted,

$$
\Delta=\left(\begin{array}{cccccccccc}
-1 & 0 & 0 & 0 & 1 & -1 & 0 & 1 & 0 & 0 \\
1 & -1 & 0 & 0 & 0 & 0 & -1 & 0 & 1 & 0 \\
0 & 1 & -1 & 0 & 0 & 0 & 0 & -1 & 0 & 1 \\
0 & 0 & 1 & -1 & 0 & 1 & 0 & 0 & -1 & 0
\end{array}\right)
$$

The analogues of the F-terms are those necessary for the vanishing of the term $\left|\left[X^{1}, X^{2}\right]\right|^{2}$ at the minimum of the classical scalar potential. These terms, of the form $\left[X^{1}, X^{2}\right]=0$ are not all independent. In fact, one can check that they can be solved in terms of six independent fields, which we denote collectively by $v_{j}, j=1 \cdots 6$. The solution can be expressed in terms of a matrix $K$, such that the original fields of the theory, which we denote by $X_{i}$, can be expressed in terms of the six independent fields $v_{j}$ as $X_{i}=\prod_{j} v_{j}^{K_{i j}}$. In this example, the (transpose of) the matrix $K$ is given by

$$
K^{t}=\left(\begin{array}{cccccccccc}
1 & 0 & 0 & 0 & 0 & 0 & -1 & -1 & 0 & 0 \\
0 & 1 & 0 & 0 & 0 & 0 & 0 & -1 & -1 & 0 \\
0 & 0 & 1 & 0 & 0 & 0 & 0 & 0 & -1 & -1 \\
0 & 0 & 0 & 1 & 0 & 0 & 1 & 1 & 1 & 0 \\
0 & 0 & 0 & 0 & 1 & 0 & 0 & 1 & 1 & 1 \\
0 & 0 & 0 & 0 & 0 & 1 & 1 & 1 & 1 & 1
\end{array}\right)
$$

We now introduce a new set of fields, $p_{\alpha}, \alpha=1, \cdots c$, which are the physical fields in the linear sigma model corresponding to this orbifold. The reason for this transformation is to avoid the singularities that may arise, due to negative entries in (35). Given the matrix $K$, we calculate its dual cone $T$, which is composed of the set of vectors dual to $K$, i.e $\vec{K} . \vec{T} \geq 0$, following the algorithm given in [14. Then, the dual cone defines our new set of fields $p_{\alpha}$, where the relation between the independent variables $v_{i}$ of the original theory and these fields is given by $v_{i}=\prod_{\alpha} p_{\alpha}^{T_{i \alpha}}$. In our case, the dual cone is given by

$$
T=\left(\begin{array}{cccccccccccc}
0 & 0 & 0 & 0 & 0 & 0 & 0 & 0 & 1 & 1 & 1 & 1 \\
0 & 0 & 0 & 0 & 0 & 1 & 1 & 1 & 0 & 0 & 0 & 1 \\
0 & 0 & 0 & 1 & 1 & 0 & 0 & 0 & 0 & 0 & 1 & 1 \\
0 & 0 & 1 & 0 & 0 & 0 & 0 & 1 & 0 & 1 & 0 & 1 \\
0 & 1 & 0 & 0 & 1 & 0 & 1 & 0 & 0 & 0 & 0 & 1 \\
1 & 0 & 0 & 1 & 0 & 1 & 0 & 0 & 1 & 0 & 1 & 0
\end{array}\right)
$$

Since the number of new fields introduced is more than the number of independent fields in the original theory, we need to introduce a new gauge group 
(corresponding to a number of $C^{*}$ actions) in order to eliminate redunduncies. We can calculate the charges of the new fields $p_{\alpha}$ under this new gauge group by using conditions of gauge invariance. Let us call the matrix denoting these charges as $V_{1}$. In addition, we can determine the matrix of charges of these new fields under the original $U(1)^{4}$, which we call $V_{2}$. This matrix $V_{2}$, which is the equivalent of the D-term in the gauge theory with the new fields, will, contain the analogues of the FI parameters. Writing the matrices without the coloumn of FI parameters, concatenating $V_{1}$ and $V_{2}$, and taking the kernel of the resulting matrix gives us the geometric data for the resolution of the singularity [19], which, in this case, is found be the matrix

$$
\mathcal{T}=\left(\begin{array}{cccc}
1 & 0 & -1 & -3 \\
0 & 1 & 2 & 5
\end{array}\right)
$$

after the elimination of all the repeated coloumns. This is the expected result for the toric data of the orbifold $\mathbb{C}^{2} / \mathbb{Z}_{5(3)}$. 1

In this example, there are no supersymmetric subsectors, and the decay of this space proceeds entirely by tachyonic deformations. In the next subsection, we will study thse decays from the point of view of the inverse toric procedure [17], which is essentially an algorithm to evaluate D-brane gauge theory configurations starting from the toric data that the brane probes, i.e the opposite process of what we have described above. For the moment, having set up the necessary conventions, let us study, in a similar fashion, the example of the orbifold $\mathbb{C}^{2} / \mathbb{Z}_{8(3)}$, in which we can use marginal deformations of the theory to split the space. We will find that the D-brane toric data, in these cases, contains a novel element: it encodes the data for a marginal deformation, in addition to the usual data for the singularity.

The analysis proceeds in complete analogy with the previous example, and we have relegated the details of the calculations in the appendix. The final result for the toric geometry data for a D-brane probing the singularity $\mathbb{C}^{2} / \mathbb{Z}_{8(3)}$ are contained in the coloumns of the matrix

$$
\mathcal{T}=\left(\begin{array}{ccccc}
1 & 0 & -1 & -1 & -3 \\
0 & 1 & 4 & 3 & 8
\end{array}\right)
$$

\footnotetext{
${ }^{1}$ By constructing invariant variables in terms of the linear sigma model fields (as elaborated in the appendix for the orbifold $\mathbb{C}^{2} / \mathbb{Z}_{8(3)}$, the equation for this singularity is given by $z^{5}=x^{2} y$ in appropriate gauge invariant variables $x, y, z$.
} 
From the analysis of the previous section, we get the following flow pattern

$$
\mathbb{C}^{2} / \mathbb{Z}_{8(3)} \rightarrow \mathbb{C}^{2} / \mathbb{Z}_{4(1)} \oplus \mathbb{C}^{2} / \mathbb{Z}_{4(-3)}
$$

In agreement with [2]. Notice that while the usual toric data for this example is given by the continuted fraction $[3,3]$ with the vectors of the toric fan being given by $(1,0),(0,1),(-1,3),(-3,8)$, the toric data obtained from the D-brane probe gives the continued fraction $[4,1,4]$ with the added vector $(-1,4)$ which corresponds to a marginal deformation by the fourth twisted sector, which, as can be checked, is a supersymmetric subsector. Hence, we see that the toric data of the D-brane gauge theory contains an additional point which is blown up, this point corresponding to a marginal deformation of the theory. Figure (2) give the toric diagram for the orbifold $\mathbb{C}^{2} / \mathbb{Z}_{8(3)}$. As we can see, the point corresponding to the marginal deformation lies on the line joining the initial and final vectors of the cone.

Continuing along the same lines, let us now proceed to analyse nother example, the non-supersymmetric orbifold $\mathbb{C}^{2} / \mathbb{Z}_{10(3)}$. The explicit matrices are not presented here due to space constraints. The final result for the toric data, from considerations of the D-brane gauge theory is

$$
\mathcal{T}=\left(\begin{array}{cccccc}
1 & 0 & -1 & -1 & -2 & -3 \\
0 & 1 & 5 & 4 & 7 & 10
\end{array}\right)
$$

Whereas the original Hirzebruch-Jung singularity in this case is given by $[4,2,2]$, we see that the toric data given by the D-brane gauge theory is $[5,1,3,2]$, with an additional point blown up, corresponding to a deformation by the fifth twisted sector, which is supersymmetric. Once again, we can obtain the split in the toric data in accordance with [2].

Let us now turn to the class of examples where the orbifolding group is of odd order. One such example is the non-supersymmetric orbifold $\mathbb{C}^{2} / \mathbb{Z}_{9(5)}$ which has marginal deformations in the third twisted sector. The result for the toric data obtained from the D-brane gauge theory is, in this example,

$$
\mathcal{T}=\left(\begin{array}{ccccc}
1 & 0 & -1 & -1 & -5 \\
0 & 1 & 3 & 2 & 9
\end{array}\right)
$$

This corresponds to the continued fraction $\frac{9}{5}=[3,1,6]$ and, comparing with the original continued fraction for this singularity, which is given by $[2,5]$, we see that the D-brane toric data once again provides us with the correct marginal 
deformation, corresponding to turning on the point $(3,-1)$. The explicit matrices for this example are presented in the appendix.

Let us mention at this point that in [6], it was argued that decays of singularities of the form (15) may not be allowed in the CFT description for large values of $l$. In the cases that we have discussed in (39) and (40), $l$ is sufficiently small, and the results are consistent with the $g_{c l}$ conjecture of HKMM. However, purely from a D-brane probe analysis, there is no way to rule out the large $l$ flows. We believe that a study of higher dimensional examples might shed more light into this apparent contradiction.

We are now ready to discuss some examples of non-supersymmetric 3-fold orbifolds. The supersymmetric 3 -folds were briefly discussed before, and as we have mentioned, they can be treated in the same way as supersymmetric 2folds, following HKMM. Let us now discuss the gauge theory data for D-branes probing 3-fold orbifolds. We will show that once again, the toric data encodes the information about the marginal deformations of the theory, as in the twofold examples. The low energy theory is again a quiver gauge theory, and one can proceed to determine the resolutions of these orbifolds along the lines of [2], using the analogues of the FI parameters as before. The calculations are entirely similar to the two-fold cases, and we will not include the details. We present some results below.

Consider first the non-supersymmetric orbifold given by $\left[\frac{1}{5}(1,1,2)\right]$. The classical D-brane world volume theory is a gauge theory $U(1)^{5}$ gauge theory in this case, and the toric data can be calculated following the procedure of [8], and is given by

$$
\mathcal{T}=\left(\begin{array}{ccccc}
1 & 0 & 0 & -1 & -2 \\
0 & 1 & 0 & 0 & -1 \\
0 & 0 & 1 & 3 & 5
\end{array}\right)
$$

This is expected, as from (31), we see that the toric fan, in this example, is generated by the one dimensional cones given by the vectors $(5,-1,-2),(0,1,0),(0,0,1)$. The vector $(1,0,0)$ corresponds to adding an internal ray with the weight vector $\zeta=\left(\frac{1}{5}, \frac{1}{5}, \frac{2}{5}\right)$, and the vector $(3,0,-1)$ is the internal ray with the weight vector $\zeta^{3}$.

Let us now go over to our next example, $\left[\frac{1}{6}(1,1,2)\right]$. There are non-isolated singularities in this case, since the rank of the orbifolding group is non-prime. We can, however, construct the D-brane gauge theory data as in the previous examples. This example is interesting, because this orbifold has marginal deformations, 
corresponding to the third twisted sector, which corresponds to $\left[\frac{1}{2}(1,1,2)\right]$ (noting that with fermions included, the integers in the brackets are defined modulo 4 , we see that this is a supersymmetric subsector). The final result for the toric data for this case is

$$
\mathcal{T}=\left(\begin{array}{cccccc}
1 & 0 & 0 & -1 & -1 & -2 \\
0 & 1 & 0 & 0 & 0 & -1 \\
0 & 0 & 1 & 3 & 4 & 6
\end{array}\right)
$$

Let us consider eq. (43) in some details. In this case, the toric fan is generated by the vectors $(6,-1,-2),(0,1,0),(0,0,1)$. The weight vector for the orbifold action, as follows from eq. (32) is $\zeta=\left(\frac{1}{6}, \frac{1}{6}, \frac{2}{6}\right)$, and the vector $(1,0,0)$ corresponds to adding the internal ray with these weights. The vector $(4,0,-1)$ is the internal ray corresponding to $\zeta^{4}$. Similarly, the vector $(3,0,-1)$ corresponds to the action of $\zeta^{3}$ on the vectors of the toric fan (note that the integers appearing in the weight vector is defined modulo 6), and the latter, as we have pointed out, is a marginal deformation. Thus, as in the $\mathbb{C}^{2}$ example, the D-brane gauge theory data corresponding to a non-supersymmetric three-fold orbifold contains an additional point, which, as before, corresponds to a marginal deformation.

As a final example, consider the orbifold $\left[\frac{1}{8}(1,1,2)\right]$, the fourth subsector of which has marginal deformations. The result for the toric data in this case is

$$
\mathcal{T}=\left(\begin{array}{cccccc}
1 & 0 & 0 & -1 & -1 & -2 \\
0 & 1 & 0 & 0 & 0 & -1 \\
0 & 0 & 1 & 4 & 5 & 8
\end{array}\right)
$$

Similar to our previous example, we see that the vector $(4,0,-1)$ corresponds to a deformation by the fourth subsector of the theory, which is a supersymmetric subsector.

In all the above three-fold orbifold examples, the methods of the previous subsection can be used to split the toric data, and study the RG flows. These can also be understood by generalising the procedure of [6]. These flows will involve perturbing by various relevant and marginal operators, as in the twofold case. We will, however, leave a complete study of the same for a future publication.

\subsection{The Inverse Toric Procedure}

Now that we have discussed examples of D-brane world-volume gauge theories for branes probing non-supersymmetric orbifolds from the toric geometry point 
of view, we can ask the question of the existance of toric duality 17 for nonsupersymmetric orbifolds. Indeed, the end point of closed string tachyon condensation in the class of examples that we have studied is always a supersymmetric orbifold, but these orbifolds have opposite supersymmetry [2] compared to the usual supersymmetric orbifolds. Hence, it is possible that we might learn something new by considering toric duals of the gauge theories that we have been considering so far.

It is well known that an interesting aspect of world volume gauge theories of D-branes probing toric singularities is the so called toric duality, first proposed in [17]. Simply stated, this duality principle states that there can be more than one D-brane gauge theory that flows in the IR to the same universality class i.e share the same toric description. This, in particular, followed from the inverse toric algorithm developed in [17] which allows one to read off the world volume gauge theory data of D-branes from the toric data of the singularity that it probes. It is a useful tool in the context of non-supersymmetric orbifolds, as we will show now. Let us start by briefly reviewing the inverse toric algorithm of [17].

The computation of the geometric data of the singularity probed by a Dbrane was developed in [19]. As we have already pointed out in the beginning of the last subsection, this method consists of concatenating the D and F-terms of the (supersymmetric) gauge theory of the D-brane world volume into a matrix that describes the dual cone of the toric variety that the brane probes. In our discussion in the previous subsections, we have carried out this procedure for nonsupersymmetric orbifolds also, and we have shown that interestingly, the result often describes a non-minimal resolution of the orbifold, with additional points in the toric diagram corresponding to marginal perturbations of the theory.

The inverse toric algorithm, on the other hand, involves embedding the original orbifold singularity into one of higher rank, and then determining the partial resolutions of the latter in order to reach the lower rank orbifold in question. It turns out that in the process, one might discover new gauge theories that are torically dual to the original one.

The above procedure is similar to the one that we followed in determining the flows of non-supersymmetric D-brane orbifold gauge theories to supersymmetric ones (or for that matter from supersymmetric gauge theories for orbifolds of higher ranks to those of lower rank). The important point to note here is that, in the language of [17], the final supersymmetric orbifold theories that are obtained from the flows can be embedded in non-supersymmetric orbifolds. Then, giving 
vev's to some of the original fields of the theory can be equivalently stated in terms of the fields of the linear sigma model corresponding to the orbifold. Knowing exactly which fields are to be resolved in order to flow from a non-supersymmetric to a supersymmetric orbifold, we can determine the conditions on the classical moduli space (corresponding to the analogues of the FI parameters) of the original theory. To set the notation, let us illustrate this with an example.

We choose the simple model of $\mathbb{C}^{2} / \mathbb{Z}_{8(3)}$. From the brane probe point of view, we have seen that the flows seen by the D-brane are those for which one can construct marginal deformations of the gauge theory. This would, in particular, correspond to choosing a subset of the analogues of the Fayet-Illiapoulos parameters and setting linear combinations of them to zero. Any such combination, provided the relevant deformation is marginal, would result in an orbifold of lower rank, starting from the parent orbifold. In the inverse toric procedure, this would imply that we resolve a certain number of points in the toric diagram in a way consistent with turning on of these parameters in the gauge theory. For this particular example, the number of physical fields was found to be 24 (see eqn. (51) of the appendix), and gauge invariant polynomials are constructed in terms of these.

The equation for the singularity can be constructed in terms of the gauge invariant parameters of the original theory. In this example, there are three gauge invariant combinations that we can form (the others being equivalent to these), and the expressions for these are presented in the appendix. As expected, when expressed in terms of the linear sigma model fields, they satisfy a relation of the form $x y=z^{4}$. We note that from the toric data of eq. (39), the toric diagram for the singularity $\mathbb{C}^{2} / \mathbb{Z}_{4(1)}$ can be embedded into that of $\mathbb{C}^{2} / \mathbb{Z}_{8(3)}$, as shown in fig. (2) and using this, we can determine the fields in the linear sigma model that need to be resolved in order to effect the flow $\mathbb{C}^{2} / \mathbb{Z}_{8(3)} \rightarrow \mathbb{C}^{2} / \mathbb{Z}_{4(1)}$ using the techniques of [17].

Equivalently, from the analysis of section 2 (following [2]), it is clear that in order to retain a $\mathbb{Z}_{4}$ symmetry at the end, we need to give vevs to four of the fields which are then resolved, and the remaining four correspond to the residual $\mathbb{Z}_{4}$ symmetry. Let us choose these to be the fields $X_{12}, X_{34}, X_{56}, X_{78}$. From the expression for these fields in terms of the linear sigma model fields (51), it can be seen that in terms of these, this resolution implies that the fields that we need to 


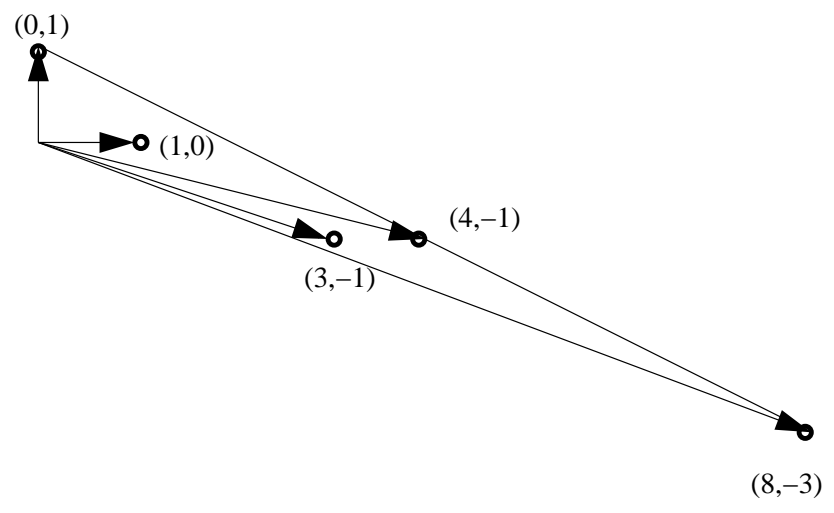

Figure 2: The toric diagram for the singularity $\mathbb{C}^{2} / \mathbb{Z}_{8(3)}$, corresponding to eq. (39). The toric diagram for $\mathbb{C}^{2} / \mathbb{Z}_{4(1)}$, which consists of the vectors $(4,-1),(1,0),(0,1)$ can be embedded into this.

retain in this process are $p_{1}, p_{2}, p_{4}, p_{6}, p_{11}, p_{15}$, and the rest are eliminated. I From this data, we can construct the reduced charge matrix of the fields by removing the relevant coloumns from the dualcone of eq. (51), and by suitably rearranging its rows so as to make sure that this reduced dualcone is appropriate for the reduced toric data, obtained after removing the points $(8,-3)$ and $(3,-1)$ from the original toric data. If we also add the coloumn containing the analogues of the FI parameters in the original charge matrix $V_{2}$ (in the notation immediately following eq. (36)), the reduced charge matrix includes these, and the final result for this matrix is

$$
Q_{\text {total }}=\left(\begin{array}{ccccccc}
1 & -1 & -1 & -1 & -1 & 1 & 0 \\
0 & 0 & -1 & 1 & 0 & 0 & \lambda_{5}+\lambda_{6} \\
0 & 0 & 0 & -1 & 1 & 0 & \lambda_{3}+\lambda_{4} \\
0 & 1 & 0 & 0 & -1 & 0 & \lambda_{1}+\lambda_{2}
\end{array}\right)
$$

Where the $\lambda \mathrm{s}$ are the analogues of the FI parameters. Let us mention that from this matrix, we can immediately read off the region of parameter space that we are dealing with. From eq. (45), it is clear that in order to effect the turning

\footnotetext{
${ }^{2}$ This was one of the problems addressed in [17]. In general, the resolution of a node of a toric diagram implies the resolution of more than one field in the linear sigma model. In the method of [17], the fields that have to be resolved are determined by the embedding of the toric diagram, in this case, they are equivalently determined by looking at the vevs given to the fields of the original theory.
} 
on of the vevs of the original fields in the theory in the manner that we have done in the previous paragraph, we need to set one of the seven FI parameters to a finite positive value (and hence remove it from the toric data), and choose the linear combinations of the other six to be zero. This choice of the classical parameter space can also be obtained directly by following the procedure of [17], i.e by performing a Gaussian row reduction on the original charge matrix, and tuning the fields so that one gives non-zero vevs to some fields while staying in the physical region of the parameter space where the FI parameters $\lambda_{i} \geq 0$. From (45), after following the procedure of [17, the charge matrix (corresponding to the quiver diagram) of the resulting gauge theory can be found to be

$$
d=\left(\begin{array}{cccccccc}
0 & 1 & -1 & 0 & 0 & 1 & -1 & 0 \\
1 & -1 & 0 & 0 & 1 & -1 & 0 & 0 \\
-1 & 0 & 0 & 1 & -1 & 0 & 0 & 1 \\
0 & 0 & 1 & -1 & 0 & 0 & 1 & -1
\end{array}\right)
$$

which is the charge matrix for the orbifold $\mathbb{C}^{2} / \mathbb{Z}_{4(1)}$ where the last row represents the trivial $U(1)$ corresponding to the centre of mass motion of the brane.

In deriving the above result, we have assumed that the first row in (45) corresponds to the F-term and the other three rows correspond to the D-terms of the final gauge theory. However, as has been pointed out in [17], the division of the matrix (45) into D and F terms is actually arbitrary. Writing the matrix (45) without specifying the FI parameters, we can make a different choice for the D and the F-terms. Let us study this in some more details. If, in (45), we chose the first two rows as specifying the F-terms, and the other two as corresponding to the D-terms, a calculation in the lines of [17] shows that we get a charge matrix

$$
d=\left(\begin{array}{cccccc}
0 & -1 & 1 & 0 & 1 & -1 \\
1 & 0 & -1 & 1 & -1 & 0 \\
-1 & 1 & 0 & -1 & 0 & 1
\end{array}\right)
$$

where, as before, we have added the trivial $U(1)$. The dual matrix $K$ of the kernel of the F-term matrix (which, in this case, is the first two rows of the matrix in (45)) is given by

$$
K=\left(\begin{array}{llllll}
0 & 0 & 0 & 1 & 1 & 2 \\
0 & 0 & 1 & 0 & 1 & 0 \\
0 & 1 & 0 & 0 & 0 & 1 \\
1 & 0 & 0 & 1 & 0 & 0
\end{array}\right)
$$


Since the nullspace of $K$ has dimension 2, we expect two relationships between the coloumns of $K$ for this theory. They are given by $\left(X_{1} X_{3} X_{6}=X_{2} X_{4} X_{5}\right)$, and $\left(X_{1} X_{5}=X_{3} X_{4}\right)$. Now, from the charge matrix in eq. (47), we can construct the gauge invariant quantities, $a=X_{1} X_{2} X_{3}, b=X_{4} X_{5} X_{6}, c=X_{2} X_{3} X_{4}, d=$ $X_{1} X_{5} X_{6}, e=X_{1} X_{2} X_{5}, f=X_{3} X_{4} X_{6}, g=X_{1} X_{3} X_{6}$ and $h=X_{2} X_{4} X_{5}$. Using the first of the relations between the coloumns, we obtain $a b=g^{2}$, which is the equation for the singularity $\mathbb{C}^{2} / \mathbb{Z}_{4(1)}$. P The same relation can be derived by considering other algebraic relations between the gauge invariant variables. For eg., it can be seen by using both the relations between the coloumns of $K$ in (48) that another possible algebraic relation between the gauge invariant variables is $d e=g^{2}$. Integrating the relations between the coloumns of $K$ to form the superpotential will in general need the introduction of new (presumably chargeless) fields in the theory, and in general non zero values of these new fields might lead us into different branches of the moduli space. We leave a detailed discussion of this issue for the future.

While the above example seems to suggest some sort of toric duality [20], with the matrix (47) corresponding to a $U(1)^{3}$ D-brane world volume gauge theory, which is different from the $U(1)^{4}$ theory of (46), let us point out a few caveats. In the non-supersymmetric case that we have been considering, our discussion is restricted to vanishing string coupling, and therefore, we cannot make a statement about the two gauge theories of (46) and (47) as being dual (that flow to a common fixed point in the IR) at this point. The example given above should be thought of as relating two gauge theories that have the same classical moduli space. Nevertheless, we believe that it might be possible to make a stronger assertion about the quantum corrections and the IR behaviour of the two theories in (46) and (47), and work is in progress in this direction.

Further, both the theories in eqs. (46) and (47) have chiral fermions and hence will have anomalies at the quantum level. Anomaly cancellation in the usual supersymmetric case can be checked following the procedure advocated in [8]. We have not performed such explicit checks here.

At this stage, let us point out that it is important in these examples that the toric data for the final (supersymmetric) singularity that we want to reach can be embedded in the original (non-supersymmetric) one. This can be done

\footnotetext{
${ }^{3}$ Note that the orbifold $\mathbb{C}^{2} / \mathbb{Z}_{4(1)}$ has the opposite supersymmetry as compared to the usual orbifold $\mathbb{C}^{2} / \mathbb{Z}_{4(-1)}$. While the equation for the latter singularity is $x y=z^{4}$, the former is given by $x y=z^{2}$, in some appropriate gauge invariant variables.
} 
when the original orbifold has marginal deformations. For the $\mathbb{C}^{2}$ orbifolds, these deformations correspond, in the toric diagram, to additional points on the edges of the toric diagram, as in fig. (2). This process of embedding is however, not possible for non-supersymmetric orbifolds without marginal deformations, and we do not expect the inverse toric procedure to be of much use in those cases. In this paper, we have studied the inverse toric algorithm for two-fold orbifolds only. It will be very interesting to carry out this analysis for the case of three-fold orbifolds.

\subsection{Weighted Projective Spaces}

Clearly, we expect the above analysis to go over to the case of higher dimensional orbifolds, of the form $\mathbb{C}^{n} / \Gamma$. Already, for $n=3$, we expect a much richer structure than two-fold orbifolds. One possible route to investigate would be the behaviour of brane probes on weighted projective spaces, considered in [5]. Consider, for example, the supersymmetric orbifold $\mathbb{C}^{3} / \mathbb{Z}_{4}$ with the action of the discrete group on the coordinates being given by

$$
\left(Z^{1}, Z^{2}, Z^{3}\right) \rightarrow\left(\omega Z^{1}, \omega Z^{2}, \omega^{2} Z^{3}\right)
$$

Where $\omega$ is a fourth root of unity. The blowup of the origin of this orbifold will

correspond to the weighted projective space $\mathbb{C P}_{1,1,2}^{2}$. A similar analysis can be done for the non-supersymmetric orbifolds in $\mathbb{C}^{3}$. One can ask if D-brane probes will be useful in studying decays of the blowups of such orbifolds. Vafa [5] has considered the mirror Landau-Ginzburg models corresponding to such weighted projective spaces. Clearly, the analogues of these are expected to be seen in the corresponding Gepner models, and it might be possible to study these decays from the geometric point of view as presented here. This will involve the construction of the analogue of a Poincare polynomial in lines with the supersymmetric case. We expect the toric geometry of brane probes to be an useful tool of analysis in such cases.

\section{Summary}

In this paper, we have carried out an investigation of the condensation of closed string tachyons in non-supersymmetric orbifold theories, in the sub-stringy regime. 
As tools, we have used the D-brane probe method developed in [2] and toric geometry methods, which are intimately related to the chiral ring techniques of [6]. We have provided a partial classification of flow patterns in orbifolds of the form $\mathbb{C}^{2} / \mathbb{Z}_{n}$ using quiver techniques. We have seen from a consideration of the D-brane gauge theory that it probes the correct singularity for the non-supersymmetric orbifolds, and, where appropriate, the toric data arising from the D-brane gauge theory provides additional points corresponding to marginal deformations.

We have also examined a few examples of three-fold orbifolds, and found that even in these cases, the toric data of the D-brane probing these singularities encodes the information about the marginal deformations by adding additional points to the toric diagram.

Further, we have applied the inverse toric algorithm to a class of non-supersymmetric orbifolds, which have marginal deformations in some of the twisted sectors. We have shown that this algorithm can be applied to these non-supersymmetric orbifolds, and we have initiated a discussion of toric duality in the same. Whereas in [20], dual D-brane gauge theories were studied for supersymmetric orbifolds, an extension of our results in this paper might give examples of such duality in orbifolds which have the opposite supersymmetry compared to the usual supersymmetric ones.

It would be interesting to further this investigation in a number of directions. Even though the probe method has limited applicability, it might prove to be useful for analysis of higher dimensional non-supersymmetric orbifolds, In particular, the inverse toric method can be used to ask questions about a more general underlying structure of toric duality. Further, as we have mentioned, a direct application of our methods can be made in the study of the decay of weighted projective spaces. Consider, for example, the model $\mathbb{P}_{1,1,2,2,2}^{4}$. Methods of [21] and [22] can be used to deduce the singularity structure for this manifold in a Landau-Ginzburg framework, and one can calculate the various forms that need to be blown up in order to resolve the singularities of this space. It would be interesting to consider this example in the light of its realisation as the blowup of a corresponding (non-supersymmetric) orbifold of the form $\mathbb{C}^{5} / \Gamma$.

Also, it would be interesting to extend this type of analysis in the case of orbifolds of product groups, for eg. of the form $\mathbb{C}^{3} / \mathbb{Z}_{m} \times \mathbb{Z}_{n}$. It follows from [9] that for such theories, the phase diagram is more complicated than those for $\mathbb{Z}_{n}$ orbifolds. It would be very interesting to study closed string tachyon condensation in these theories, by toric geometry methods. We leave these issues for a future 
publication.

\section{Acknowledgements}

We would like to thank S. Mukhopadhyay and K. Ray for useful discussions and for collaboration during the initial part of this project. We would also like to thank K. S. Narain, G. Thompson, M. S. Narasimhan, D. Goswami and T. E. Venkata Balaji for helpful discussions.

\section{Appendix}

\subsection{Toric data for the singularity $\mathbb{C}^{2} / \mathbb{Z}_{8(3)}$}

The Matrix $K^{t}$ is given by

$$
K^{t}=\left(\begin{array}{cccccccccccccccc}
1 & 0 & 0 & 0 & 0 & 0 & 0 & 0 & 0 & -1 & -1 & -1 & -1 & -1 & 0 & 0 \\
0 & 1 & 0 & 0 & 0 & 0 & 0 & 0 & 0 & 0 & -1 & -1 & -1 & -1 & -1 & 0 \\
0 & 0 & 1 & 0 & 0 & 0 & 0 & 0 & 0 & 0 & 0 & -1 & -1 & -1 & -1 & -1 \\
0 & 0 & 0 & 1 & 0 & 0 & 0 & 0 & 0 & 1 & 1 & 1 & 0 & 0 & 0 & 0 \\
0 & 0 & 0 & 0 & 1 & 0 & 0 & 0 & 0 & 0 & 1 & 1 & 1 & 0 & 0 & 0 \\
0 & 0 & 0 & 0 & 0 & 1 & 0 & 0 & 0 & 0 & 0 & 1 & 1 & 1 & 0 & 0 \\
0 & 0 & 0 & 0 & 0 & 0 & 1 & 0 & 0 & 0 & 0 & 0 & 1 & 1 & 1 & 0 \\
0 & 0 & 0 & 0 & 0 & 0 & 0 & 1 & 0 & 0 & 0 & 0 & 0 & 1 & 1 & 1 \\
0 & 0 & 0 & 0 & 0 & 0 & 0 & 0 & 1 & 1 & 1 & 1 & 1 & 1 & 1 & 1
\end{array}\right)
$$

The dual cone is generated by

$$
\left(\begin{array}{llllllllllllllllllllllll}
0 & 0 & 0 & 0 & 0 & 0 & 0 & 0 & 0 & 0 & 0 & 0 & 0 & 0 & 0 & 0 & 1 & 1 & 1 & 1 & 1 & 1 & 1 & 1 \\
0 & 0 & 0 & 0 & 0 & 0 & 0 & 0 & 0 & 0 & 1 & 1 & 1 & 1 & 1 & 1 & 0 & 0 & 0 & 0 & 0 & 0 & 1 & 1 \\
0 & 0 & 0 & 0 & 0 & 0 & 1 & 1 & 1 & 1 & 0 & 0 & 0 & 0 & 0 & 1 & 0 & 0 & 0 & 0 & 1 & 1 & 0 & 1 \\
0 & 0 & 0 & 0 & 0 & 1 & 0 & 0 & 0 & 1 & 0 & 0 & 0 & 1 & 1 & 0 & 0 & 1 & 1 & 1 & 0 & 0 & 0 & 1 \\
0 & 0 & 0 & 0 & 1 & 0 & 0 & 0 & 1 & 0 & 0 & 1 & 1 & 0 & 0 & 0 & 0 & 0 & 0 & 1 & 0 & 1 & 1 & 1 \\
0 & 0 & 0 & 1 & 0 & 0 & 0 & 1 & 0 & 0 & 0 & 0 & 0 & 0 & 1 & 1 & 0 & 0 & 1 & 0 & 1 & 0 & 1 & 1 \\
0 & 0 & 1 & 0 & 0 & 0 & 0 & 0 & 0 & 1 & 0 & 0 & 1 & 1 & 0 & 1 & 0 & 1 & 0 & 0 & 0 & 1 & 0 & 1 \\
0 & 1 & 0 & 0 & 0 & 0 & 0 & 1 & 1 & 1 & 0 & 1 & 0 & 0 & 1 & 0 & 0 & 0 & 0 & 1 & 0 & 0 & 0 & 1 \\
1 & 0 & 0 & 0 & 0 & 0 & 1 & 0 & 0 & 0 & 1 & 0 & 0 & 0 & 0 & 1 & 1 & 0 & 0 & 0 & 1 & 1 & 1 & 0
\end{array}\right)
$$


The charge matrix under the original $U(1)^{7}$ is

$$
Q=\left(\begin{array}{cccccccccccccccc}
-1 & 0 & 0 & 0 & 0 & 0 & 0 & 1 & -1 & 0 & 0 & 0 & 0 & 1 & 0 & 0 \\
1 & -1 & 0 & 0 & 0 & 0 & 0 & 0 & 0 & -1 & 0 & 0 & 0 & 0 & 1 & 0 \\
0 & 1 & -1 & 0 & 0 & 0 & 0 & 0 & 0 & 0 & -1 & 0 & 0 & 0 & 0 & 1 \\
0 & 0 & 1 & -1 & 0 & 0 & 0 & 0 & 1 & 0 & 0 & -1 & 0 & 0 & 0 & 0 \\
0 & 0 & 0 & 1 & -1 & 0 & 0 & 0 & 0 & 1 & 0 & 0 & -1 & 0 & 0 & 0 \\
0 & 0 & 0 & 0 & 1 & -1 & 0 & 0 & 0 & 0 & 1 & 0 & 0 & -1 & 0 & 0 \\
0 & 0 & 0 & 0 & 0 & 1 & -1 & 0 & 0 & 0 & 0 & 1 & 0 & 0 & -1 & 0
\end{array}\right)
$$

From these, the toric data given in (38) can be calculated. The gauge invariant combinations in terms of the physical fields (corresponding to the coloumns of the dual cone in (51) are given by

$$
\begin{aligned}
X & =X_{12} X_{23} X_{34} X_{45} X_{56} X_{67} X_{78} X_{81} \\
& =p_{2} \cdots p_{7} p_{8}^{3} p_{9}^{3} p_{10}^{4} p_{11} p_{12}^{3} p_{13}^{3} p_{14}^{3} p_{15}^{4} p_{16}^{4} p_{17} p_{18}^{3} p_{19}^{3} p_{20}^{4} p_{21}^{3} p_{22}^{4} p_{23}^{4} p_{24}^{8} \\
Y & =Y_{14} Y_{47} Y_{72} Y_{25} Y_{58} Y_{83} Y_{36} Y_{61} \\
& =p_{1}^{8}\left(p_{2} \cdots p_{7}\right)^{3} p_{8} p_{9} p_{10}^{4} p_{11}^{3} p_{12} p_{13} p_{14} p_{15}^{4} p_{16}^{4} p_{17}^{3} p_{18} p_{19} p_{20}^{4} p_{21} p_{22}^{4} p_{23}^{4} \\
Z & =X_{12} Y_{25} X_{56} Y_{61} \\
& =p_{1}^{2} p_{2} \cdots p_{9} p_{10}^{2} p_{11} \cdots p_{14} p_{15}^{2} p_{16}^{2} p_{17} p_{18} p_{19} p_{20}^{2} p_{21} p_{22}^{2} p_{23}^{2} p_{24}^{2}
\end{aligned}
$$

\subsection{Toric data for the singularity $\mathbb{C}^{2} / \mathbb{Z}_{9(5)}$}

For this example, the matrix $K^{t}$ is given by

$$
\left(\begin{array}{cccccccccccccccccc}
1 & 0 & 0 & 0 & 0 & 0 & 0 & 0 & 0 & 0 & -1 & -1 & -1 & -1 & 0 & 0 & 0 & 0 \\
0 & 1 & 0 & 0 & 0 & 0 & 0 & 0 & 0 & 0 & 0 & -1 & -1 & -1 & -1 & 0 & 0 & 0 \\
0 & 0 & 1 & 0 & 0 & 0 & 0 & 0 & 0 & 0 & 0 & 0 & -1 & -1 & -1 & -1 & 0 & 0 \\
0 & 0 & 0 & 1 & 0 & 0 & 0 & 0 & 0 & 0 & 0 & 0 & 0 & -1 & -1 & -1 & -1 & 0 \\
0 & 0 & 0 & 0 & 1 & 0 & 0 & 0 & 0 & 0 & 0 & 0 & 0 & 0 & -1 & -1 & -1 & -1 \\
0 & 0 & 0 & 0 & 0 & 1 & 0 & 0 & 0 & 0 & 1 & 1 & 1 & 1 & 1 & 0 & 0 & 0 \\
0 & 0 & 0 & 0 & 0 & 0 & 1 & 0 & 0 & 0 & 0 & 1 & 1 & 1 & 1 & 1 & 0 & 0 \\
0 & 0 & 0 & 0 & 0 & 0 & 0 & 1 & 0 & 0 & 0 & 0 & 1 & 1 & 1 & 1 & 1 & 0 \\
0 & 0 & 0 & 0 & 0 & 0 & 0 & 0 & 1 & 0 & 0 & 0 & 0 & 1 & 1 & 1 & 1 & 1 \\
0 & 0 & 0 & 0 & 0 & 0 & 0 & 0 & 0 & 1 & 1 & 1 & 1 & 1 & 1 & 1 & 1 & 1
\end{array}\right)
$$


The dual cone, $T$ in this example, is generated by

$$
\left(\begin{array}{lllllllllllllllllllllll}
0 & 0 & 0 & 0 & 0 & 0 & 0 & 0 & 0 & 0 & 0 & 0 & 0 & 0 & 0 & 0 & 0 & 0 & 1 & 1 & 1 & 1 & 1 \\
0 & 0 & 0 & 0 & 0 & 0 & 0 & 0 & 0 & 0 & 0 & 0 & 0 & 0 & 1 & 1 & 1 & 1 & 0 & 0 & 0 & 0 & 1 \\
0 & 0 & 0 & 0 & 0 & 0 & 0 & 0 & 0 & 0 & 1 & 1 & 1 & 1 & 0 & 0 & 0 & 0 & 0 & 0 & 0 & 0 & 1 \\
0 & 0 & 0 & 0 & 0 & 0 & 0 & 1 & 1 & 1 & 0 & 0 & 0 & 0 & 0 & 0 & 0 & 0 & 0 & 0 & 0 & 1 & 1 \\
0 & 0 & 0 & 0 & 0 & 1 & 1 & 0 & 0 & 0 & 0 & 0 & 0 & 0 & 0 & 0 & 0 & 1 & 0 & 0 & 1 & 0 & 1 \\
0 & 0 & 0 & 0 & 1 & 0 & 0 & 0 & 0 & 0 & 0 & 0 & 0 & 1 & 0 & 0 & 1 & 0 & 0 & 1 & 0 & 0 & 1 \\
0 & 0 & 0 & 1 & 0 & 0 & 0 & 0 & 0 & 0 & 0 & 0 & 1 & 0 & 0 & 1 & 0 & 0 & 0 & 0 & 0 & 1 & 1 \\
0 & 0 & 1 & 0 & 0 & 0 & 0 & 0 & 0 & 1 & 0 & 1 & 0 & 0 & 0 & 0 & 0 & 1 & 0 & 0 & 0 & 0 & 1 \\
0 & 1 & 0 & 0 & 0 & 0 & 1 & 0 & 1 & 0 & 0 & 0 & 0 & 1 & 0 & 0 & 0 & 0 & 0 & 0 & 0 & 0 & 1 \\
1 & 0 & 0 & 0 & 0 & 1 & 0 & 1 & 0 & 0 & 1 & 0 & 0 & 0 & 1 & 0 & 0 & 1 & 1 & 0 & 1 & 1 & 0
\end{array}\right)
$$

Finally, the charge matrix of the fields under the original $U(1)^{8}$ is

$$
\left(\begin{array}{cccccccccccccccccc}
-1 & 0 & 0 & 0 & 0 & 0 & 0 & 0 & 1 & -1 & 0 & 0 & 0 & 1 & 0 & 0 & 0 & 0 \\
1 & -1 & 0 & 0 & 0 & 0 & 0 & 0 & 0 & 0 & -1 & 0 & 0 & 0 & 1 & 0 & 0 & 0 \\
0 & 1 & -1 & 0 & 0 & 0 & 0 & 0 & 0 & 0 & 0 & -1 & 0 & 0 & 0 & 1 & 0 & 0 \\
0 & 0 & 1 & -1 & 0 & 0 & 0 & 0 & 0 & 0 & 0 & 0 & -1 & 0 & 0 & 0 & 1 & 0 \\
0 & 0 & 0 & 1 & -1 & 0 & 0 & 0 & 0 & 0 & 0 & 0 & 0 & -1 & 0 & 0 & 0 & 1 \\
0 & 0 & 0 & 0 & 1 & -1 & 0 & 0 & 0 & 1 & 0 & 0 & 0 & 0 & -1 & 0 & 0 & 0 \\
0 & 0 & 0 & 0 & 0 & 1 & -1 & 0 & 0 & 0 & 1 & 0 & 0 & 0 & 0 & -1 & 0 & 0 \\
0 & 0 & 0 & 0 & 0 & 0 & 1 & -1 & 0 & 0 & 0 & 1 & 0 & 0 & 0 & 0 & -1 & 0
\end{array}\right)
$$

From this data, one obtains (41). 


\section{References}

[1] A. Sen, "Non-BPS States and Branes in String Theory," hep-th/9904207

[2] A. Adams, J. Polchinski and E. Silverstein, "Don't panic! Closed String Tachyons in ALE spacetimes", JHEP 0110 (2001) 029. hep-th/0108075

[3] P. Aspinwall, "Resolution of Orbifold Singularities in String Theory," In B. Greene, S. T. Yau (eds)Mirror symmetry II, 355-379, hep-th/9403123i.

[4] M. Douglas, "Topics in D-geometry," Class. Quant. Grav. 17 (2000) 1057, hep-th/9910170

[5] C. Vafa, "Mirror Symmetry and Closed String Tachyon Condensation," hep-th/0111051

[6] J. Harvey, D. Kutasov, E. Martinec and G. Moore, "Localized tachyons and RG flows," hep-th/0111154

[7] J. David, M. Gutperle, M. Headrick and S. Minwalla, "Closed String Tachyons Codensation on Twisted Circles,"tt hep-th/0111212

"Tachyon Mass, C Function and Counting Localized Degrees of Freedom," hep-th/0202097

R. Rabadan, J. Simon, "M Theory Lift Brane Anti-brane Systems and Localised Closed String Tachyons," JHEP 0205 (2002) 045

A. Basu, "Localised Tachyons and the G(cl) Conjecture," hep-th/0204247

[8] M. Douglas and G. Moore, "D-branes, quivers and ALE instantons,"hep-th/9603167

[9] E. Witten, "Phases of $\mathrm{N}=2$ Theories in Two Dimensions," Nuclear Physics B 403, (1993) 159, hep-th/9301042.

[10] K. Hori, C. Vafa, "Mirror Symmetry," hep-th/0002222

[11] D-E. Diaconescu, M. Douglas, "D-branes on Stringy Calabi-Yau Manifolds," hep-th/0006224

[12] L. Dixon, D. Friedan, E. Martinec, S. Shenker, "The Conformal Field Theory of Orbifolds," Nucl. Phys. B 282 (1987) 13.

[13] L. Dixon, "Some Worldsheet Properties of Superstring Compactifications, on Orbifolds or Otherwise," Lectures given at the ICTP summer workshop, Trieste, 1987. 
[14] W. Fulton, "Introduction to Toric Varieties,", Ann. Math. Studies, 131, Princeton University Press, 1993.

[15] P. Aspinwall, B. Greene, D. Morrison, "Calabi-Yau Moduli Space, Mirror Manifolds and Spacetime Topology Change in String Theory," Nucl. Phys. B 416, (1994), 414 hep-th/9309097.

[16] B. Greene, "String Theory on Calabi-Yau Manifolds," hep-th/9702155.

[17] B. Feng, A. Hanany, Y-H. He, "D-brane Gauge Theories from Toric Singularities and Toric Duality," Nucl. Phys. B 595 (2001) 165.

[18] T. Oda, "Convex Bodies and Algebraic Geometry," Speringer-Verlag, 1988.

[19] M. Douglas, B. Greene, D. Morrison, "Orbifold Resolution by D-branes," Nucl. Phys. B 506 (1997) 84.

[20] C. Beasley, M. R. Plesser, "Toric Duality is Seiberg Duality," JHEP 0112 (2001) 001 hep-th/0109053

B. Feng, A. Hanany, Y-H He, "Toric Duality as Seiberg Duality and Brane Diamonds," JHEP 0112 (2001) 035, hep-th/0109063.

[21] S. Roan, S-T. Yau, "On Ricci Flat 3-Fold," Acta Math. Sinica (New Series) 3 (1987) 256.

[22] C. Vafa, "String Vacua and Orbifoldized L-G Models," Mod. Phys. Lett. A 4 (1989) 1169. 